DOI:10.2478/rrlm-2019-0025

\title{
Laboratory medicine in the era of precision medicine - dream or reality?
}

\author{
Minodora Dobreanu ${ }^{1,2^{*}}$, Oana Roxana Oprea ${ }^{1,2}$ \\ 1. The Advanced Medical and Pharmaceutical Research Center, University of Medicine, Pharmacy, \\ Sciences and Technology of Târgu Mureș, Romania; 2. Clinical Laboratory of Emergency Districtual \\ Hospital Targu Mures, Romania
}

Received: 30 ${ }^{\text {th }}$ March 2019; Accepted: 11 th April 2019; Published: $15^{\text {th }}$ April 2019

\section{Precision medicine or personalized medicine?}

Precision medicine (PM) is a concept that approaches the customization of healthcare by tailoring medical decisions, treatments or products to the individual. Personalized medicine is an older mien with a similar sense to PM (they are frequently used interchangeably) which was proposed to be replaced by PM because it could be misinterpreted to imply that treatment would be developed uniquely for each individual (the concept does not mean production of unique medication for a subject). The aim in PM is to identify which approaches will be effective for which subjects, based on genetic, life style, environmental factors, etc. It means the ability to classify individuals into subgroups that differ in their predisposition to a particular condition they may develop, or in their response to a particular treatment (1). Therapeutic or preventive actions can then be focused on those who will benefit most, sparing costs and side effects for those subjects who will not.

In 2015 president Obama announced a government funded initiative that aimed to accelerate progress of PM, with a near-term focus on better cancer prevention and treatment and a lon- ger-term aim applicable to the whole range of health and disease (2). A longitudinal cohort of one million people was planned for embarking on who would voluntarily participate with extensive characterization of biological specimens (metabolic biomarkers, cells, DNA, RNA, etc.), physiological and behavioral/ environmental data link to their electronic health records to build a comprehensive scientific base. Growth of digital health as a source of continuous and rich personal data (due to ubiquitous adoption of electronic medical records), dramatic decline in cost and increase in throughput of DNA sequencing in the last 15 years, after human genome was first sequenced, would facilitate building of a new "pool of gene data" as well as genome-phenom interrogation to generate significant knowledge for the network of scientists. However, the ambitious project is suspended due to lack of financial support nowadays.

\section{Scope of Laboratory Medicine in the era of Precision Medicine - where are we today?}

PM ecosystem ideally links patients, research and/or clinical laboratories and healthcare providers. Laboratory medicine (LM) is an indis-

*Corresponding author: Minodora Dobreanu, The University of Medicine, Pharmacy, Sciences and Technology of Târgu Mureș, Romania. E-mail: minodora.dobreanu@umfst.ro 
pensable discipline generating $70 \%$ of information used in healthcare processes which will play an essential role to support the implementation of PM in clinical settings.

In diagnostic testing precision there are different levels of efficacy: we have good current evidence for diagnostic accuracy and technical efficacy of medical laboratory methods (3), but we need more research for evaluation of patient outcome, therapeutic and societal efficacy. For technical efficacy we have excellent achievements; a "temple of laboratory standardization" is based on reference methods and materials, accredited reference laboratories, traceable reference intervals and decision limits, appropriately organized quality control and proficiency test programs, error measurement and targets for uncertainty (4, 5). The application of the analytical performance specification can be modulated depending on its use. Decision limit is established according to clinical performance aim (it depends on the purpose of the test: screening, diagnosis, detection of recurrence, etc.).

Even if most biomarkers in use are surrogate markers, they are "windows" into pathophysiology of particular diseases, decision support for diagnosis, prognosis and risk stratification. "Gold standard" biomarkers like creatinine clearance/ eGFR and Cystatin $\mathrm{C}$ in kidney disease, cardiac troponins (cTn) in myocardial damage, natriuretic peptides (NT-proBNP, BNP) in hemodynamic stress and heart failure, HbAlc for diagnosis and monitorization in diabetes, are widely used in spite of their "imperfection" in some subpopulations (eGFR overdiagnoses renal disease in elderly people, cTn needs lower limit of decision for myocardial injury in women, natriuretic peptides overdiagnose heart failure in people with chronic kidney disease, inadequacy of the use of $\mathrm{HbA} 1 \mathrm{c}$ as the only indicator of glycemic control in diabetic subjects with cardiovascular disease). Diagnostic thinking is focused nowadays on assessing test results produced by medical labora- tories against population-based reference ranges, expected in $95-99 \%$ of presumed healthy individuals (previously called "normal" or "physiological" ranges) (6). In 2008, the International Federation of Clinical Chemistry and Laboratory Medicine (IFCC-LM) revised the recommendations for establishing reference ranges and current guidelines were published by the Clinical and Laboratory Standards Institute (CLSI C28A3) (7). Producing their own reference ranges is not an easy task for laboratories and nowadays the need to revise the concept is emerging - the theory of reference ranges is back in fashion. This is the main reason which caused alternative methods to be found and indirect methodologies to be encouraged by IFCC Committee on Reference Intervals and Decision Limits (8). Starting 2013, the Romanian Journal of Laboratory Medicine has published several articles proposing Hoffmann technique $(9,10)$ for determinations of reference intervals for hemoglobin, serum total magnesium and calcium in Romanian children $(11,12)$, and decision limits for serum uric acid associated with cardiovascular morbidity and metabolic syndrome in this issue (13).

On the other hand, by using the reference ranges concept for interpretation of test results there is a grey area where superposition of values from healthy and ill subject exists. For instance, PSA, which is still worldwide accepted biomarker for prostate cancer screening, at a threshold of $4 \mathrm{ng} /$ $\mathrm{ml}$ (recommended for most ELIA techniques) has excellent negative predictive value $(>98 \%)$ to rule-out healthy individuals, but overdiagnoses prostate cancer (less than $80 \%$ of subjects are rule-in after biopsy) in the range of 4-10 $\mathrm{ng} /$ $\mathrm{ml}$ due to large overlapping with benign pathologies; in symptomatic men with PSA in this range and negative digital rectal examination, cut-offs for biopsy could be raised in different subpopulations in order to avoid men to undergo unnecessary biopsy (14). Due to poor specificity of total PSA, a PSA in the range of "grey zone" in 
asymptomatic men aged 50 and over triggers reflex testing of free PSA (fPSA) or complex PSA (cPSA), which could reduce the number of unnecessary biopsies. It is hoped that research into emerging markers will eventually validate more accurate investigations such as PSA glycoforms, TMPRSS2:ERG fusion gene, PCA3 gene, miRNAs, circulating tumor cells, androgen receptor variants, and PTEN gene (15).

A second example for the evolution of clinical decision limit concept would be assessment of the patient with suspected myocardial infarction (MI) based on serum concentrations of cTn. cTn are the biomarkers which define MI, recommended to both rule-out and rule-in myocardial injury patients. Evolution history of definitions of MI has an epidemiologic era than a clinical approach using evidence of elevated serum cTn at least one value above the $99^{\text {th }}$ percentile upper reference limit (URL). Due to the fact that in chronic myocardial injury cTn frequently exceed $99^{\text {th }}$ percentile URL, the fourth universal definition of acute MI (AMI) recently published (16) is based on kinetics of cTn: delta troponin is the rate of changes (\%) between two cTn results taken in the same patient within a 3-4 hour time period; a delta troponin $>+/-30 \%$, with one value for $\mathrm{cTn}>14 \mathrm{ng} / \mathrm{L}$ ( $>9 \mathrm{ng} / \mathrm{L}$ for women) in 2-3 hours, is consistent with an AMI in the right clinical setting. Detection of a rise and/ or fall of cTn concentrations is an essential early component along with symptomatology and ECG changes. On the other hand, cTn is a quantitative marker of cardiomyocyte injury, but not a screening tool.

Working with the reference ranges will remain the way for interpretation of laboratory results many years from now. However, future works should be directed to the real contribution of the reference ranges and limits to better define their position in medical decision-making. It must not be forgotten that there are primarily evidence-based clinical decision limits (like symptoms, mortality, rehospitalization), but also that frontiers between health and pathology are grey zones influenced by many biological and environmental factors. This kind of approach is absolutely necessary to ensure optimal use of laboratory tests.

\section{Multimarker approach in precision medicine}

Using advanced laboratory technology and a variety of "-omics" LM is prone to discover and evaluate analytical performance and clinical value of novel biomarkers. Multiplex technologies are versatile and flexible to enable simultaneous screening of extended panels of biomarkers with reasonable costs, reduced time and labor over traditional assays, integrating laboratory functions on a chip or on a suspension array technology.

A modest proportion of discovered biomarkers are implemented in routine medical practice, mainly due to poor specificity in some settings and poor sensitivity in others. Filling the gap between biomarker discovery and meaningful clinical use (change of the patient outcome) is slow and challenging.

In many pathologies (especially in cerebral-cardiometabolic, oncologic and infectious diseases) targeted multiple biomarker panels is considered a superior approach of precision medicine for early diagnosis, risk stratification, decision support, prognostication, monitorization and/ or optimization of therapies, compared with single biomarkers.

Complete blood count (CBC) derived parameters such as Mean Corpuscular Volume (MCV), mean corpuscular hemoglobin concentration (MCHC), and mean corpuscular hemoglobin $(\mathrm{MCH})$ are used routinely in clinical practice in the diagnosis of different type of anemias or hemoglobinopathy. Recently other/new CBC derivative parameters like platelet-to-lymphocyte 
ratio (PL/LY), neutrophil-to-lymphocyte ratio $(\mathrm{PMN} / \mathrm{LY})$ or red blood cell distribution width to lymphocyte count ratio (RDW/LY) have been proposed to be used for other purposes/pathologies and were verified also in the Romanian subpopulation as predictors for coronary chronic total occlusion in patients presenting with ST-segment elevation MI - PL/LY (17) to support proper clinical decision making in patients with high risk non-muscle invasive bladder cancer - PMN/ LY (18), or aiming at maximizing selection of those with the likelihood of celiac disease who shall benefit from confirmatory serologic tests (serum IgA anti-tissue transglutaminase 2 and anti-endomysial antibodies and duodenal biopsy) - RDW/LY (19).

Patient stratification in cardio-cerebrovascular and metabolic disorders is on big demand nowadays; of the most challenging in biomarker development are prognostic markers associated with left ventricular dysfunction or remodeling (GDF-15, metalloproteinases MMP, TIMP and Gal-3 are promising markers). One of the recent challenging multiple biomarker screening (255 plasma proteins) was performed from the ARISTOTLE trial in order to identify novel prognostic markers for major bleeding in patients with atrial fibrillation on oral anticoagulation (20).

In our population we measured a panel of five peripheral biomarkers for neuronal damage, inflammation, and fibrinolysis and we found that D-Dimers and sTNFR-1 have increased discrimination ability to classify poor functional outcome at 3 months after ischemic stroke (21).

\section{Importance of molecular/genetic diagnostic in precision medicine - from bench to bedside}

Improving the effectiveness of health care is assured by a combination of revolutionary technologies and evolutionary practice which accelerates transformation of future medicine.
Precision medicine is no longer just a promise for the future. Every day, it is moving more and more into clinical practice. Molecular diagnostic is the fastest growing field within laboratory testing; the term "molecular" covers an array of techniques including proteins, DNA, RNA, miRNA, mRNA, the tendency being to make genomics-based diagnostic a routine. This is transforming health care by offering new tests for patient stratification, typically relying on the ability to identify individuals with specific genetic elements that define a disease or therapeutic efficiency. These results may be used not only to select proper treatment modalities, but also to identify subjects who may benefit from the preventive approach.

The ability to provide precision medicine services to patients in routine clinical practice depends on the availability of quick and accurate molecular tests; molecular testing continues to become faster and cheaper (feasible even for point-of-care variants). Nowadays, a long list of molecular diagnostic tests is used in laboratories for unexplained disorders; many of them are analytically validated, some of them are still expected to prove clinical utility and/or public health validity.

\section{A. Identification of genetic variants as the basis of disease}

Detection of single nucleotide polymorphism (SNP) $1691 \mathrm{G}>\mathrm{A}$ of $F 5$ gene (factor V Leiden, FVL - the most common hereditary hypercoagulable disorder among Eurasians), 20210G $>$ A of F2(prothrombin) gene and MTHFR (methylenetetrahydrofolate reductase) C677T and A1298C polymorphisms in patients with deep venous thrombosis; in young Romanian women homozygosity for FVL and for MTHFR A1298C polymorphisms increase the risk for venous thrombosis (22) 
Mutations in any of the three genes $L D L R$ (low density lipoprotein receptor), $A P O B$ (apolipoprotein $B$ ) and PCSK9 (Proprotein convertase subtilisin/kexin type 9) are known to cause autosomal dominant familial hypercholesterolemia, FH (the most prevalent genetic disorder in the general population and a major risk factor for atherosclerosis); cascade screening in descendings from patients with premature acute myocardial infarction permit prophylactic approach. However, FH is underevaluated and probably underestimated, mainly due to the relatively low proportion $(\sim 40 \%)$ of monogenic mutations found in subjects with clinical diagnosis of $\mathrm{FH}$ (according to DUTCH LIPID CLINIC NETWORK DIAGNOSTIC, MEDPED or Simon Broome criteria). For the rest of the subjects, classified according to clinical scores, the etiology of hypercholesterolemic phenotype is very probably polygenic - multiple SNPs-Scores could help. The ability to select those subjects with a positive clinical diagnosis who have a monogenic or a polygenic etiology is a model of the application of genomic data using cholesterol-lowering drugs with different efficiency and costs.

Homozygosity for ApoE2/E2 (apolipoprotein E) is correlated with one of the most severe mixt dyslipidemia (type III dyslipidemia with persistent intermediary density lipoproteins IDL) and homozygosity for ApoE4/E4 - with Alzheimer disease.

Either a homozygous DR4-DQ8 genotype or a heterozygous DR4-DQ8 and HLA DR3 risk genotype are used to stratify risk of developing type 1 diabetes.

Identification of HLA-DQ2 and -DQ8 heterodimer are performed to exclude the possibility of having celiac disease in subjects with serology and/or inconclusive biopsy, or to eliminate repeated serology testing in family members.

Mutations $-13910 \mathrm{C}>\mathrm{T}$ and/or $-22018 \mathrm{G}>\mathrm{A}$ of MCM6 (minichromosome Maintenance Com- plex Component 6) gene are found in patients with lactose intolerance.

HLA-B27 alleles are checked to rule-out ankylosing spondylitis and other autoimmune arthritis types.

Molecular diagnostics are essential to diagnose and to monitor patients with some infectious diseases, especially in immunocompromised subjects (influenza virus-specific strains as H1N1, HIV, HCV, CMV, Chlamydia, etc.), or genotypes with oncogenic potential (HPV16, -18, HTLV-I, HHV8, i.e.).

\section{B. Drug-gene testing for more precise medications - pharmacogenomics}

Pharmacogenomics is the study of how genes determine a person's reactivity to particular drugs. Efforts have been made to apply pharmacogenomics testing to improve patient care in many specialties. The main purpose is identification of people who are likely to benefit from, and people who may require careful monitoring in order to establish a tailored dose of medication, identification of people who are likely to be at increased risk for serious side effects and should not receive the treatment; no longer "one size fits all"! Here are some examples:

Efficiency of treatment with HMG-CoA (3-hydroxy-3-methylglutaryl coenzyme A reductase) inhibitors in hypercholesterolemic patients, but also side/toxic effects (like myopathy, hepatotoxicity) are influenced by polymorphism in cytochrome enzyme CYP3A4 and CYP2C9*2 genes (according to the type of statin used); the most important interactions appear in combination with CYP3A4 activators (carbamazepine, barbiturates, rifampicin) or inhibitors (macrolides, azole antifungals, amiodarone, foods such as grapefruit juice) which require dose adjustment. The SLCO1B1 (solute carrier organic anion transporter family member $1 \mathrm{~B} 1$, also known as $O A T P 1 B$ ) gene encodes the organic anion he- 
patocellular transporter OATP1B of a variety of endogenous compounds and drugs. A SLCO1B1 variant has been associated with an increased risk of myopathy during simvastatin therapy due to altered pharmacokinetics of this drug.

Subjects carrying $A$ allele of the vitamin K epoxide reductase complex subunit 1 VKORCl and/ or $C Y P 2 C 9 * 2$ and/or $* 3$ gene polymorphisms are called "poor metabolizers" of K vitamin inhibitors; variant enzymes resulting is less active than normal enzymes, decreasing the metabolism of coumarin derivatives which results in an associated bleeding risk and need for a lower dose of acenocoumarol.

$20-65 \%$ of individuals in different populations have loss-of-function CYP2C19 gene polymorphism leading to a poor metabolization of clopidogrel and determine insufficient in vivo activation of pro-drugs P2Y12 inhibitors and poor inhibition of platelet reactivity despite treatment. In a Romanian group with acute coronary syndromes and non-cardiogenic ischemic stroke we found $19 \%$ of clinically non-responders to this drug (in line with Western European populations); this occurred especially in individuals who were carriers of $C Y P 2 C 19 * 2$ and/ or $C Y P 2 C 19 * 3$ allele, even if only $10 \%$ of them were homozygotes and $29 \%$ heterozygotes (23). Other patients possess increase of function polymorphism in the enzyme CYP $2 C 19 * 17$ gene that makes them rapid metabolizers, but the prevalence is much lower than of poor metabolizers.

Most of the beta-blockers used for treatment of heart failure, angina, and hypertension are metabolized in the liver by CYP2D6 ("debrisoquin -oxidase") enzyme with more than 100 isoforms; normal function alleles are CYP2D6*1, *2, *33, *35. In about $10 \%$ of the population, no function (the most common include $C Y P 2 D 6 * 3, * 4, * 5$ and $* 6$ ) or decreased function alleles (the most common are CYP2D6*9, $* 10, * 17, * 29$ and $* 41)$ were found. In addition, drugs like propafenone, paroxetine, quinidine, and fluoxetine inhibit CYP2D6 activity; " $C Y$ $P 2 D 6$ poor metabolizers" have 5-10 times higher prevalence of side effects after metoprolol administration.

A possible molecular mechanism of multidrug resistance epilepsy involves the silent polymorphisms of drug efflux transporter of antiepileptic drugs $A B C B 1$ gene (ATP-binding cassette subfamily B member 1), also known as MDR 1 (multidrug resistance 1) gene. In a recently published Romanian study, $A B C B 1-129 \mathrm{~T}>\mathrm{C}$ showed a significant association with the sub-therapeutic plasmatic concentration of antiepileptic drugs, but the frequency of patients who did not reach therapeutic concentrations was reduced comparatively with the frequency of non-responsive patients - other mechanism of resistance being possibly involved (24).

Hypersensitivity reaction to abacavir is strongly associated with the presence of the $H L A-B * 5701$ allele; carriers of this allele should avoid the drug entirely to eliminate a serious adverse event.

\section{Genomic tests for more precise management of cancer}

Traditional tumor biomarkers like PSA, CEA, AFP, CA15-3, CA 19-9, CA 27-29, CA-125 have defective sensibility and specificity especially because most of them are performed in peripheral blood, but they are still routinely used in cancer treatment decisions mainly due to affordable cost and to the fact that sophisticated markers are not easily available (25). Of the nucleic-acid based tumor biomarkers, the US Food and Drug Administrations (FDA) currently acknowledge less than 25 markers for diagnostic/prognostic and predictive purpose (Table 1) (26). Due to the complexity of the topic it needs a deeper approach in another issue.

Analytical validation is only the first part in the process of in vitro diagnostic (IVD) tumor 
Table 1. Diagnostic, prognostic, and predictive nucleic-acid based tumor biomarkers FDA approved (26)

\begin{tabular}{lccc}
\hline Cancer type & Predictive markers & Diagnostic markers & Prognostic markers \\
\hline Breast & HER2, ER/PR* & MG, CK19 & HER2, TOP2A \\
& HER2 in CTC ** & & 58-, 70-gene RNA ex- \\
& & pression profile
\end{tabular}

\begin{tabular}{|c|c|c|c|}
\hline Prostate & & PCA3 & tPSA \\
\hline $\begin{array}{l}\text { Gastro-intestinal stromal } \\
\text { tumor }\end{array}$ & KIT mutation* & & \\
\hline Colorectal & KRAS mutations* & $\begin{array}{l}\text { BMP3 and NDRG4, } \\
\text { KRAS, } \beta \text {-actin }\end{array}$ & \\
\hline $\begin{array}{l}\text { Esophago-gastric } \\
\text { adenocarcinoma }\end{array}$ & HER2* & & \\
\hline Acute Myeloid Leukemia & & & EGR1 \\
\hline $\begin{array}{l}\text { Acute Promyelocytic } \\
\text { Leukemia }\end{array}$ & PML-RAR $\alpha^{*}$ & & \\
\hline Chronic Myeloid Leukemia & BCR-ABL * & & \\
\hline $\begin{array}{l}\text { B-cell Chronic } \\
\text { Lymphocytic Leukemia }\end{array}$ & & 12p11.1-q11 & $\begin{array}{c}\text { TP53, ATM, } \\
\text { 13q14.3 deletion } \\
\text { (D13S319), } \\
\text { trisomy } 12(\mathrm{D} 12 \mathrm{Z} 3)\end{array}$ \\
\hline
\end{tabular}

\begin{tabular}{lc}
\hline NSCLC & $\begin{array}{c}\text { EGFR (HER1)* } \\
\text { ALK* }\end{array}$ \\
\hline Lung adenocarcinoma & $\begin{array}{c}\text { BRAF*, HER2*, EG- } \\
\text { FR(HER1)*, KRAS* } \\
\text { ALK* }\end{array}$ \\
\hline Skin melanoma & BRAF V600* \\
\hline Ovary & \multicolumn{2}{c}{ BRCA1 and 2 } \\
\hline Bladder & Aneuploidy of chr 3, \\
& \\
\hline
\end{tabular}

ALK, anaplastic lymphoma kinase; ATM, serine/threonine kinase; BRCA1/2 tumor suppressor genes; EGFR, epidermal growth factor receptor; ER, Estrogen receptor; ERK, extracellular-signal-regulated kinases; HER2: Epidermal growth factor receptor 2; NSCLC, non-small cell lung cancer; PML, Promyelocytic leukemia gene; PR, progesterone receptor; RAR $\alpha$, retinoic acid receptor-alpha; CTC-circulating tumor cells $\mathrm{MG}$, mammaglobin; PSA, prostate specific antigen; TOP2A, topoisomerase II alpha; chr. chromosome

* In clinical use

** Under clinical evaluation

biomarkers development. Clinical utility validation and clinical implementation are even more challenging in making markers suitable for clinical diagnostic/prognostic and treatment decision (27).

\section{The importance of liquid biopsies for precision medicine}

Conventional prenatal diagnostic tests for chromosomal abnormalities currently involve an amniocentesis procedure or chorionic villus sam- 
pling with risk of inducing miscarriage. On the other hand, rare fetal cells originating from the placenta can be found in a mother's bloodstream (about one in a million). Using modern microfluidic technology, it is now possible to isolate these cells from the mother's white blood cells or even cell-free fetal DNA/RNA which has recently revolutionized the field of aneuploidy and microdeletion screening in pregnancy by enabling non-invasive prenatal testing.

Traditional methods, such as flow cytometry, detect extremely rare subpopulations (i.e. circulating tumor cells CTC) which allows early detection of cancer and treatment effectiveness.

Circulating extracellular RNAs with origins across all cell types have the potential to serve as biomarkers for a wide range of medical conditions. Identifying the specific tissue where the RNA originates along with its molecular structure and its abundance in the circulation has enormous potential for detecting disease processes and discovering new abnormalities. Exciting new technologies and thousands of listing examples of various miRNAs that have been identified as potential biomarkers, outbreak current scientific publications. In previous issues of the Romanian Journal of Laboratory Medicine some miRNA-30c and miRNA-190 were presented with prognostic abilities in patients with colon and lung cancer, but also a series of miRNAs that can serve as biomarkers for the evaluation of the critically ill polytrauma patient respectively (28-30). However, there is still a long way to go for the analytical and clinical validation of these classes of biomarkers.

\section{What are the challenges the precision medicine initiative is facing?}

Precision Medicine initiative needs modern technologies; some of them are in the early stages of development and some others are still too expensive to become part of routine healthcare (e.g. DNA sequencing); however, guidance for analytical validation of Next Generation Sequencing (NGS) - based on IVDs intended to aid in the diagnosis of suspected germline diseases, have already been released by FDA (31).

Reimbursement from insurance companies is also likely to become an issue. Healthcare providers will need to know more about genetic and molecular tests, collaboration among different experts will be necessary for better understanding of how that information is relevant to prevent or treat disease, to be used in clinical practice.

Databases to efficiently store and analyze a large amount of information, standardization of data collection are other challenging tasks.

The PM initiative also raises legal and ethical components - patients' privacy and protection of confidentiality of health information will be critical aspects.

Digitalisation of society and of health care system will cause the end of Laboratory Medicine as we know it nowadays. Laboratory medicine will play a key role in the development of precision medicine, not only from a technical point of view, but also by adopting new responsibilities in the clinical translation of complex information generated in a rapidly changing healthcare environment.

\section{Authors' contribution}

MD (Conceptualization; Investigation; Methodology; Project administration; Resources; Supervision; Writing - original draft; Writing - review \& editing)

ORO (Formal analysis; Methodology; Visualization; Writing - review \& editing)

\section{Conflict of interest}

None to declare. 


\section{References}

1. https://ghr.nlm.nih.gov/primer/precisionmedicine/precisionvspersonalized

2. https://obamawhitehouse.archives.gov/ blog/2015/01/30/precision-medicine-initiative-data-driven-treatments-unique-your-own-body

3. Sandberg S, Fraser CG, Horvath AR, Jansen R, Jones $\mathrm{G}$, Oosterhuis W, et al. Defining analytical performance specifications: Consensus Statement from the 1st Strategic Conference of the European Federation of Clinical Chemistry and Laboratory Medicine. Clin Chem Lab Med 2015;53(6):833-5. DOI: 10.1515/cclm-20150067

4. Elizabeta Topic, Nora Nikolac, Mauro Panteghini, Elvar Theodorsson, Gian Luca Salvagno, Marijana Miler, et al How to assess the quality of your analytical method?, Clin Chem Lab Med 2015; 53(11): 1707-18. DOI: 10.1515/cclm-2015-0869

5. Federica Braga, Mauro Panteghini, Verification of in vitro medical diagnostics (IVD) metrological traceability: Responsibilities and strategies. Clinica Chimica Acta. May 2014;432:55-61. DOI: 10.1016/j.cca.2013.11.022

6. Siest G, Henny J, Grasbeck R, Wilding P, Petitclerc C, Queralto JM, et al. The theory of reference values: an unfinished symphony. Clin Chem Lab Med 2013;51:4764. DOI: $10.1515 / \mathrm{cclm}-2012-0682$

7. CLSI. Defining, establishing, and verifying reference intervals in the clinical laboratory; approved guideline - third edition CLSI document C28-A3. Wayne, PA: Clinical and Laboratory Standards Institute, 2008.

8. Graham R.D. Jones, Rainer Haeckel, Tze Ping Loh, Ken Sikaris, Thomas Streichert, Alex Katayev, et al, on behalf of the IFCC Committee on Reference Intervals and Decision Limits, Indirect methods for reference interval determination - review and recommendations, Clin Chem Lab Med. 2019;57(1):20-29. DOI: 10.1515/ cclm-2018-0073

9. Hoffmann RG. Statistics in the practice of medicine. JAMA.1963;185:864-873. DOI: 10.1001/ jama.1963.03060110068020

10. Katayev A, Balciza C, Seccombe WD, Establishing Referance Intervals for Clinical Laboratory Test Results -is there a better way? Am J Clin Pathol, 2010, 130, 180-186. DOI: 10.1309/AJCPN5BMTSF1CDYP

11. Totan M, Gligor FG, Bojita M, Grigore C, Grigore C. Determining hemoglobin reference values in children and teenagers from Sibiu area. Rev Romana Med Lab. 2013;21(1):39-45. DOI: 10.2478/rrlm-2013-0016

12. Antonescu E, Szakács J, Totan M. Determination of reference intervals for total calcium and magnesium specific for children and adolescents in Sibiu area. Rev Romana Med Lab. 2016;24(3):347-50. DOI: 10.1515/ rrlm-2016-0030

13. Catalin Codreanu, Horatiu Popoviciu, Elena Rezus, Corina Delia Mogosan, Ioannis Gardikiotis, Claudiu Costinel Popescu, Reference interval and upper limit of normal for serum uric acid - an evidence-based approach on Romanian population using an a posteriori method, Rev Romana Med Lab. 2019;27(2):147-158. DOI: 10.2478/rrlm-2019-0019

14. Agnihotri S, Mittal RD, Kapoor R, Mandhani A. Raising cut-off value of prostate specific antigen (PSA) for biopsy in symptomatic men in India to reduce unnecessary biopsy. Indian J Med Res. 2014;139:851-6.

15. Xavier Filella,Esther Fernández-Galan,Rosa Fernández Bonifacio, Laura Foj Emerging biomarkers in the diagnosis of prostate cancer. Pharmgenomics Pers Med. 2018 May;1611:83-94. DOI: 10.2147/PGPM.S136026

16. Thygesen K, Alpert JS, Jaffe AS, Chaitman BR, Bax JJ, Morrow DA, et al. ESC Scientific Document Group, Fourth universal definition of myocardial infarction (2018). Eur Heart J. 2019 Jan 14;40(3):237-269. DOI: 10.1093/eurheartj/ehy462

17. Hadadi L, Sus I, Lakatos EK, Serban RC, Scridon A, Demjen Z, et al. Platelet indices and platelet-to-lymphocyte ratio predict coronary chronic total occlusion in patients with acute ST-elevation myocardial infarction. Rev Romana Med Lab. 2015;23(4):407-14. DOI: 10.1515/rrlm-2015-0041

18. Martha O, Porav-Hodade D, Bălan D, Tătaru OS, Sin A, Chibelean CB, et al. Easily Available Blood Test Neutrophil-To-Lymphocyte Ratio Predicts Progression in High-Risk Non-Muscle Invasive Bladder Cancer. Rev Romana Med Lab. 2017;25(2):181-9. DOI: 10.1515/ rrlm-2017-0016

19. Balaban DV, Popp A, Beata A, Vasilescu F, Jinga M. Diagnostic accuracy of red blood cell distribution width-to-lymphocyte ratio for celiac disease. Rev Romana Med Lab. 2018;26(1):45-50. DOI: 10.2478/rrlm2018-0040

20. Siegbahn A, Hijazi Z, Lindback J, Oldgren J, Pol T, Alexander JH, et al - Screening for novel prognostic biomarkers associated with major bleeding by proxim- 
ity extension assay in petients with atrial fibrillation on oral anticoagulation: insights from the ARISTOTLE trial, abstract ESC Congress, Aug 2018.

21. Hutanu A, Iancu M, Balasa R, Maier S, Dobreanu M - Predicting functional outcome of ischemic stroke patients in Romania based on plasma CRP, sTNFR-1, D-Dimers, NGAL and NSE measured using a biochip array, Acta Pharmacologica Sinica 2018: 39(7): 122836. DOI: 10.1038 /aps.2018.26

22. Daraban AM, Trifa AP, Popp RA, Botezatu D, Șerban $\mathrm{M}$, Uscatescu V, et al. Thrombophilia genetic testing in Romanian young women with acute thrombotic events: role of Factor V Leiden, Prothrombin G20210A, MTHFR C677T and A1298C polymorphisms. Rev Romana Med Lab. 2016;24(3):291-305. DOI: 10.1515/rrlm2016-0032

23. Mărginean A, Bănescu C, Moldovan V, Scridon A, Mărginean M, Bălaşa R, et al. The impact of CYP2C19 loss-of-function polymorphisms, clinical, and demographic variables on platelet response to clopidogrel evaluated using impedance aggregometry. Clin Appl Thromb. 2017; 23:255-65. DOI: $10.1177 / 1076029616629211$

24. Sabin O, Bocșan IC, Trifa A, Major ZZ, Heghes SC, Brusturean Bota E, et al. Correlation between ABCB1 gene polymorphisms, antiepileptic drug concentrations and treatment response. Rev Romana Med Lab. 2018;26(4):479-87. DOI: 10.2478/rrlm-2018-0012
25. Selleck MJ, Senthil M, Wall NR. Making Meaningful Clinical Use of Biomarkers. Biomark Insights. 2017. 12:1177271917715236. DOI: $10.1177 / 1177271917715236$

26. https://www.fda.gov/medicaldevices/productsandmedicalprocedures/invitrodiagnostics/ucm330711.htm, accessed on april 2019

27. Goossens N, Nakagawa S, Sun X, Hoshida Y Cancer biomarker discovery and validation, Transl Cancer Res. 2015 Jun; 4(3): 256-269. DOI: 10.3978/j.issn.2218676X.2015.06.04

28. Bin L, Zhang M, Lixia L, Aimin Z, Hua Y, Yanhong $\mathrm{S}$, et al. Down-regulation of miRNA-30c predicts poor prognosis in Colorectal Cancer patients. Rev Romana Med Lab. 2016;24(4):369-75. DOI: 10.1515/rrlm2016-0037

29. Gao LW, Wang GL. miR-190, CDK1, MCM10 and NDC80 predict the prognosis of the patients with lung cancer. Rev Romana Med Lab. 2019;27(1):15-24. DOI: 10.2478/rrlm-2019-0001

30. Bedreag OH, Rogobete AF, Cradigati CA, Sarandan M, Nartita R, Horhat FG, et al. A novel evaluation of microvascular damage in critically ill polytrauma patients by using circulating microRNAs. Rev Romana Med Lab. 2016;24(1):21-30. DOI: 10.1515/rrlm-2016-0015

31. https://www.fda.gov/downloads/MedicalDevices/DeviceRegulationandGuidance/GuidanceDocuments/ UCM509838.pdf, accessed on april 2019 